\title{
Bracketing the solutions of an ordinary differential equation with uncertain initial conditions
}

\author{
Thomas Le Mézo, Luc Jaulin and Benoît Zerr
}

ENSTA-Bretagne, LabSTICC, 2 rue François Verny, 29806 Brest, France.

\begin{abstract}
In this paper, we present a new method for bracketing (i.e., characterizing from inside and from outside) all solutions of an ordinary differential equation in the case where the initial time is inside an interval and the initial state is inside a box. The principle of the approach is to cast the problem into bracketing the largest positive invariant set which is included inside a given set $\mathbb{X}$. Although there exists an efficient algorithm to solve this problem when $\mathbb{X}$ is bounded, we need to adapt it to deal with cases where $\mathbb{X}$ is unbounded.
\end{abstract}

https://doi.org/10.1016/j.amc.2017.07.036

Keywords: Abstract interpretation, ODE, Infinity, Interval computation, Dynamical systems

\section{Introduction}

In this paper, we deal with a dynamical system $\mathcal{S}$ defined by the following state equation:

$$
\dot{\mathbf{x}}(t)=\mathbf{f}(\mathbf{x}(t))
$$

where $\mathbf{x}(t) \in \mathbb{R}^{n}$ is the state vector and $\mathbf{f}: \mathbb{R}^{n} \mapsto \mathbb{R}^{n}$ is the evolution function of $\mathcal{S}$. Denote by $\varphi_{\mathrm{f}}$ the flow map of the system. This means that if at time $t_{0}$, the initial state vector is $\mathbf{x}_{0}$, then the solution of the state equation is

$$
\mathbf{x}(t)=\varphi_{\mathbf{f}}\left(t-t_{0}, \mathbf{x}_{0}\right) .
$$

In this paper, we consider that the initial state $\mathbf{x}_{0}$ is not known exactly. More precisely, $\mathbf{x}_{0}$ belongs to a box $\left[\mathbf{x}_{0}\right]$ of $\mathbb{R}^{n}$. Two problems will be treated.

Problem 1. Forward reachable set. We define the forward reachable set [2, 21, 16] as

$$
\mathbb{F}_{\left[\mathbf{x}_{0}\right]}^{+}=\left\{\mathbf{x}_{a} \mid \exists \mathbf{x}_{0} \in\left[\mathbf{x}_{0}\right], \exists t \geq 0, \mathbf{x}_{a}=\varphi_{\mathbf{f}}\left(t, \mathbf{x}_{0}\right)\right\} .
$$

Email address: thomas.le_mezo@ensta-bretagne.org, luc.jaulin@ensta-bretagne.fr, benoit.zerr@ensta-bretagne.fr (Thomas Le Mézo, Luc Jaulin and Benoît Zerr) 
The problem that we will consider is to bracket the set $\mathbb{F}_{\left[\mathbf{x}_{0}\right]}^{+}$which means that we want to characterize this set from inside and from outside. This set can be interpreted as an approximation of all solutions of (1), except that we loose the dependency with respect to $t$.

Problem 2. Positive graph. For a fixed, $t_{0}$ and $\mathbf{x}_{0}$, the positive graph of the solution of (1) corresponds to the set [3]

$$
\mathbb{G}_{t_{0}, \mathbf{x}_{0}}^{+}=\left\{\left(t, \mathbf{x}_{a}\right) \mid t \geq t_{0}, \mathbf{x}_{a}=\varphi_{\mathbf{f}}\left(t-t_{0}, \mathbf{x}_{0}\right)\right\}
$$

We still assume that $\mathbf{x}_{0} \in\left[\mathbf{x}_{0}\right]$ but also, we consider that the initial time $t_{0}$ is uncertain and is only known to belong to the interval $\left[t_{0}\right]$. In this context, we define the positive graph as the set

$$
\begin{aligned}
& \mathbb{G}_{\left[t_{0}\right],\left[\mathbf{x}_{0}\right]}^{+}=\left\{\left(t, \mathbf{x}_{a}\right)\left|\exists t_{0} \in\left[t_{0}\right], \exists \mathbf{x}_{0} \in\left[\mathbf{x}_{0}\right]\right|\right. \\
& \left.t \geq t_{0}, \mathbf{x}_{a}=\varphi_{\mathbf{f}}\left(t-t_{0}, \mathbf{x}_{0}\right)\right\},
\end{aligned}
$$

which can be interpreted as the solution of the state equation with uncertain initial state and time. Similarly to Problem 1, we want to bracket the set $\mathbb{G}_{\left[t_{0}\right],\left[\mathbf{x}_{0}\right]}^{+}$from inside and outside.

Our objective is to find a unique algorithm able to find a guaranteed inner and outer approximation of the sets $\mathbb{F}_{\left[\mathbf{x}_{0}\right]}^{+}$and $\mathbb{G}_{\left[t_{0}\right],\left[\mathbf{x}_{0}\right]}^{+}[9]$. Some existing approaches use guaranteed integration [6, 20, 23] to bracket those sets [7]. For efficiency reasons we will propose in this paper, a guaranteed approach based on interval computation [13, 9] and constraint networking [14] that do not use guaranteed integration. The main difference with existing approaches is that bisections will take place both in the time space and the state space, which makes the method both Eulerian and Lagrangian [15. This increases the complexity of the method but allows us to have a better control on the accuracy of the results.

\section{Main results}

This section shows that both problems proposed in Section 1 can be expressed as the computation of the largest positive invariant set [10] which is included inside a given set $\mathbb{X}$.

A set $\mathbb{A}$ is positive invariant for the system (1) if for any trajectory $\mathbf{x}(\cdot)$, we have

$$
\mathbf{x}(0) \in \mathbb{A}, t \geq 0 \Longrightarrow \mathbf{x}(t) \in \mathbb{A} .
$$

Given a set $\mathbb{X}$, we denote by $\operatorname{Inv}^{+}(\mathbf{f}, \mathbb{X}$ ), the largest subset of $\mathbb{X}$ (with respect to the inclusion) which is positive invariant. The largest set exists and is unique, due to the fact that the set of positive invariant sets is a complete lattice with respect to the inclusion (e.g., the union or the intersection between two positive invariant sets is positive invariant). From [3], we know that

$$
\mathbf{x}_{a} \in \operatorname{Inv}^{+}(\mathbf{f}, \mathbb{X}) \Leftrightarrow \varphi_{\mathbf{f}}\left([0, \infty], \mathbf{x}_{a}\right) \subset \mathbb{X}
$$


As a consequence, $\operatorname{Inv}^{+}(\mathbf{f}, \mathbb{X})$ can be defined in two different manners

$$
\begin{aligned}
\operatorname{Inv}^{+}(\mathbf{f}, \mathbb{X}) & =\bigcup\{\mathbb{A} \in \mathcal{P}(\mathbb{X}) \mid \mathbb{A} \text { is positive invariant }\} \\
& =\quad\left\{\mathbf{x}_{a} \in \mathbb{R}^{n} \mid \varphi_{\mathbf{f}}\left([0, \infty], \mathbf{x}_{a}\right) \subset \mathbb{X}\right\}
\end{aligned}
$$

where $\mathcal{P}(\mathbb{X})$ is the power set of $\mathbb{X}$.

The two following theorems show that our two sets $\mathbb{F}_{\left[\mathbf{x}_{0}\right]}^{+}$and $\mathbb{G}_{\left[t_{0}\right],\left[\mathbf{x}_{0}\right]}^{+}$can be defined in terms of positive invariant sets.

Theorem 1: We have

$$
\mathbb{F}_{\left[\mathbf{x}_{0}\right]}^{+}=\mathbb{R}^{n} \backslash \operatorname{Inv}^{+}\left(-\mathbf{f}, \mathbb{R}^{n} \backslash\left[\mathbf{x}_{0}\right]\right)
$$

where $\backslash$ is the set theoretic difference operator (i.e., $\mathbb{A} \backslash \mathbb{B}=\{\mathbf{x} \in \mathbb{A} \mid \mathbf{x} \notin \mathbb{B}\}$ ) and $-\mathbf{f}$ is the opposite of $\mathbf{f}($ i.e., $\forall \mathbf{x},-\mathbf{f}(\mathbf{x})+\mathbf{f}(\mathbf{x})=\mathbf{0})$.

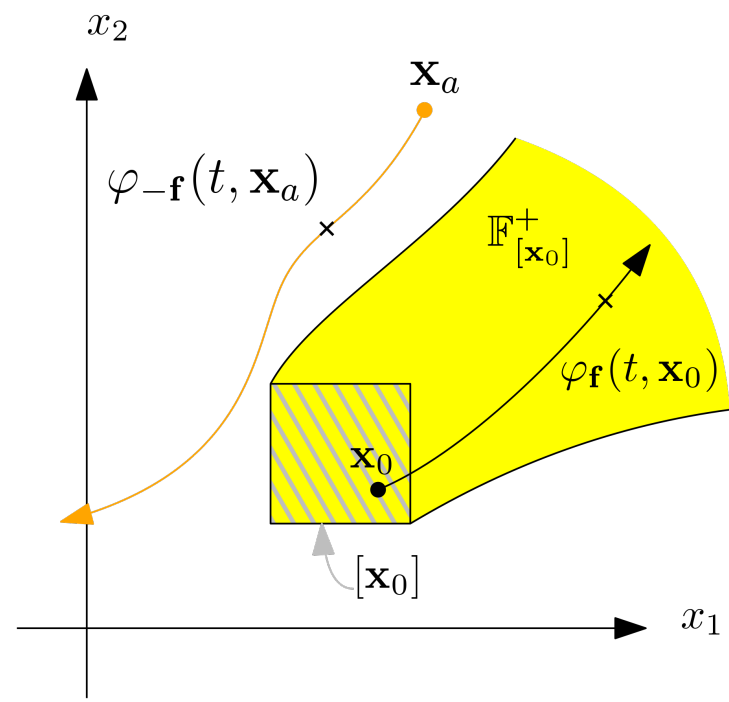

Figure 1: Illustration of the set $\mathbb{F}_{\left[\mathbf{x}_{0}\right]}^{+}$. The orange backward trajectory is outside $\mathbb{F}_{\left[\mathbf{x}_{0}\right]}^{+}$since it never reaches $\left[\mathbf{x}_{0}\right]$. The black trajectory is included in $\mathbb{F}_{\left[\mathbf{x}_{0}\right]}^{+}$.

Proof. Take an element $\mathbf{x}_{a}$ of $\operatorname{Inv} v^{+}\left(-\mathbf{f}, \mathbb{R}^{n} \backslash\left[\mathbf{x}_{0}\right]\right)$, as illustrated by Figure 1 , we have

$$
\begin{array}{cc} 
& \mathbf{x}_{a} \in \operatorname{Inv}^{+}\left(-\mathbf{f}, \mathbb{R}^{n} \backslash\left[\mathbf{x}_{0}\right]\right) \\
\Leftrightarrow & \boldsymbol{\varphi}_{-\mathbf{f}}\left([0, \infty], \mathbf{x}_{a}\right) \subset \mathbb{R}^{n} \backslash\left[\mathbf{x}_{0}\right] \\
\Leftrightarrow & \forall t \geq 0, \boldsymbol{\varphi}_{-\mathbf{f}}\left(t, \mathbf{x}_{a}\right) \in \mathbb{R}^{n} \backslash\left[\mathbf{x}_{0}\right] \\
\Leftrightarrow & \forall t \geq 0, \boldsymbol{\varphi}_{-\mathbf{f}}\left(t, \mathbf{x}_{a}\right) \notin\left[\mathbf{x}_{0}\right] \\
\Leftrightarrow & \neg\left(\exists t \geq 0, \boldsymbol{\varphi}_{-\mathbf{f}}\left(t, \mathbf{x}_{a}\right) \in\left[\mathbf{x}_{0}\right]\right) \\
\Leftrightarrow & \neg\left(\exists \mathbf{x}_{0} \in\left[\mathbf{x}_{0}\right], \exists t \geq 0, \boldsymbol{\varphi}_{-\mathbf{f}}\left(t, \mathbf{x}_{a}\right)=\mathbf{x}_{0}\right) \\
\Leftrightarrow & \neg\left(\exists \mathbf{x}_{0} \in\left[\mathbf{x}_{0}\right], \exists t \geq 0, \boldsymbol{\varphi}_{\mathbf{f}}\left(t, \mathbf{x}_{0}\right)=\mathbf{x}_{a}\right) \\
\Leftrightarrow & \neg\left(\mathbf{x}_{a} \in \mathbb{F}_{\left[\mathbf{x}_{0}\right]}^{+}\right) .
\end{array}
$$




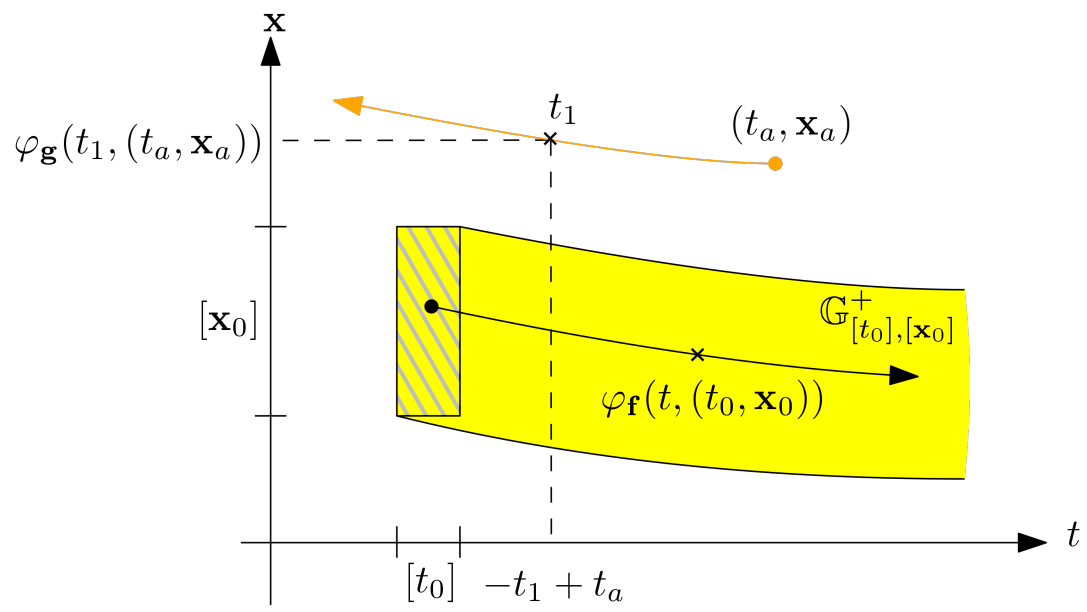

Figure 2: Illustration of the set $\mathbb{G}_{\left[t_{0}\right],\left[\mathbf{x}_{0}\right]}^{+}$. The orange trajectory is outside $\mathbb{G}_{\left[t_{0}\right],\left[\mathbf{x}_{0}\right]}^{+}$since it never reaches $\left[t_{0}\right] \times\left[\mathbf{x}_{0}\right]$. The black trajectory is included in $\mathbb{G}_{\left[t_{0}\right],\left[\mathbf{x}_{0}\right]}^{+}$.

As a consequence, the two sets $\mathbb{F}_{\left[\mathbf{x}_{0}\right]}^{+}$and $\operatorname{Inv}^{+}\left(-\mathbf{f}, \mathbb{R}^{n} \backslash\left[\mathbf{x}_{0}\right]\right)$ are complementary.

Theorem 2: We have

$$
\mathbb{G}_{\left[t_{0}\right],\left[\mathbf{x}_{0}\right]}^{+}=\mathbb{R}^{n} \backslash \operatorname{Inv}^{+}(\mathbf{g}, \mathbb{Z})
$$

where

$$
\mathbf{g}(t, \mathbf{x})=\left(\begin{array}{c}
-1 \\
-\mathbf{f}(t, \mathbf{x})
\end{array}\right), \mathbb{Z}=\mathbb{R}^{n+1} \backslash\left[t_{0}\right] \times\left[\mathbf{x}_{0}\right]
$$

Proof. Take an element $\left(t_{a}, \mathbf{x}_{a}\right)$ of $\operatorname{Inv}^{+}(\mathbf{g}, \mathbb{Z})$, as illustrated by Figure 2 we have:

$$
\begin{array}{cc} 
& \left(t_{a}, \mathbf{x}_{a}\right) \in \operatorname{Inv}^{+}(\mathbf{g}, \mathbb{Z}) \\
\Leftrightarrow & \boldsymbol{\varphi}_{\mathbf{g}}\left([0, \infty],\left(t_{a}, \mathbf{x}_{a}\right)\right) \subset \mathbb{Z} \\
\Leftrightarrow & \forall t_{1} \geq 0, \boldsymbol{\varphi}_{\mathbf{g}}\left(t_{1},\left(t_{a}, \mathbf{x}_{a}\right)\right) \in \mathbb{Z} \\
\Leftrightarrow & \forall t_{1} \geq 0, \boldsymbol{\varphi}_{\mathbf{g}}\left(t_{1},\left(t_{a}, \mathbf{x}_{a}\right)\right) \notin\left[t_{0}\right] \times\left[\mathbf{x}_{0}\right] \\
\Leftrightarrow & \forall t_{1} \geq 0,\left(\begin{array}{c}
-t_{1}+t_{a} \\
\boldsymbol{\varphi}_{-\mathbf{f}}\left(t_{1}, \mathbf{x}_{a}\right)
\end{array}\right) \notin\left[t_{0}\right] \times\left[\mathbf{x}_{0}\right] \\
\Leftrightarrow & \forall t_{1} \geq 0,-t_{1}+t_{a} \notin\left[t_{0}\right] \vee \boldsymbol{\varphi}_{-\mathbf{f}}\left(t_{1}, \mathbf{x}_{a}\right) \notin\left[\mathbf{x}_{0}\right] .
\end{array}
$$

Taking the contraposite, we get

$$
\begin{gathered}
\left(t_{a}, \mathbf{x}_{a}\right) \notin \operatorname{Inv}^{+}(\mathbf{g}, \mathbb{Z}) \\
\Leftrightarrow \quad\left\{\begin{array}{c}
\exists t_{1} \geq 0,-t_{1}+t_{a} \in\left[t_{0}\right] \\
\wedge \boldsymbol{\varphi}_{-\mathbf{f}}\left(t_{1}, \mathbf{x}_{a}\right) \in\left[\mathbf{x}_{0}\right] \\
\exists t_{1}, t_{1} \geq 0,-t_{1}+t_{a} \in\left[t_{0}\right] \\
\wedge \exists \mathbf{x}_{0} \in\left[\mathbf{x}_{0}\right], \boldsymbol{\varphi}_{-\mathbf{f}}\left(t_{1}, \mathbf{x}_{a}\right)=\mathbf{x}_{0} .
\end{array}\right.
\end{gathered}
$$


If define $t_{0}=t_{a}-t_{1}$, we get

$$
\begin{gathered}
\Leftrightarrow\left\{\begin{array}{c}
\left(t_{a}, \mathbf{x}_{a}\right) \notin \operatorname{Inv}^{+}(\mathbf{g}, \mathbb{Z}) \\
\exists t_{0}, t_{a}-t_{0} \geq 0, t_{0} \in\left[t_{0}\right] \\
\wedge \exists \mathbf{x}_{0} \in\left[\mathbf{x}_{0}\right], \boldsymbol{\varphi}_{-\mathbf{f}}\left(t_{a}-t_{0}, \mathbf{x}_{a}\right)=\mathbf{x}_{0}
\end{array}\right. \\
\Leftrightarrow \quad\left\{\begin{array}{c}
\exists t_{0} \in\left[t_{0}\right], t_{a}-t_{0} \geq 0, \exists \mathbf{x}_{0} \in\left[\mathbf{x}_{0}\right] \\
\boldsymbol{\varphi}_{-\mathbf{f}}\left(t_{a}-t_{0}, \mathbf{x}_{a}\right)=\mathbf{x}_{0}
\end{array}\right. \\
\Leftrightarrow\left\{\begin{array}{r}
\exists t_{0} \in\left[t_{0}\right], t_{a}-t_{0} \geq 0, \exists \mathbf{x}_{0} \in\left[\mathbf{x}_{0}\right], \\
\boldsymbol{\varphi}_{\mathbf{f}}\left(t_{a}-t_{0}, \mathbf{x}_{0}\right)=\mathbf{x}_{a} \\
\left(t_{a}, \mathbf{x}_{a}\right) \in \mathbb{G}_{\left[t_{0}\right],\left[\mathbf{x}_{0}\right]}^{+} .
\end{array}\right.
\end{gathered}
$$

We have thus proved that $\operatorname{Inv}^{+}(\mathbf{g}, \mathbb{Z})$ and $\mathbb{G}_{\left[t_{0}\right],\left[\mathbf{x}_{0}\right]}^{+}$are two complementary sets.

\section{Bracketing the largest invariant set}

Given a set $\mathbb{X} \subset \mathbb{R}^{n}$, the search set, which is bounded, we will show in this part that the set $\operatorname{Inv}^{+}(\mathbf{f}, \mathbb{X})$ can be bracketed from inside and outside using the algebra of mazes. By combining the inner [19] and the outer [18] approach, which were used separately before and used mazes algebra, we provide an efficient method to bracket the set $\operatorname{Inv}^{+}(\mathbf{f}, \mathbb{X})$. Indeed, less bisections of the state space will be required because of the simultaneous characterization from inside and outside.

Maze. We will now recall briefly the main ideas behind mazes. The difficulty to work with trajectories is that the dimension of the trajectory space is infinite. Therefore we need a mathematical object to grasp and work with trajectories: the mazes. Mazes was introduced using the framework of Constraint Network theory to address this problem. A constraint network [14] is composed of a set of variables, constraints and domains. Here, the variables are the paths of $\mathbb{R}^{n}$ that are consistent with (1) and the unique constraint is "the path should start from the initial condition $\left[\mathbf{x}_{0}\right]$ ". Mazes are a type of domains that allow to enclose paths, they are composed of:

- A paving $\mathcal{P}$ which covers $\mathbb{X}$,

- A polygon in each box $[\mathbf{p}]$ of $\mathcal{P}$ that encloses all the possible trajectories inside the box,

- Doors on the boundary of each box [p] that allow trajectories to leave or enter inside polygons. In our implementation, doors are union polygons (one per face of $[\mathbf{p}]$ ). We have two types of doors: output doors from which the trajectories are allowed to leave the box $[\mathbf{p}]$, and the input doors from which they are allowed to enter inside $[\mathbf{p}]$.

Figure 3 shows a representation of a part of a maze where leaving doors are painted blue and entering doors are painted red for box $\left[\mathbf{x}_{4}\right]$.

From the unique constraint, we can deduce two contractors that will be applied on doors and polygons [4, 1, 5]: 


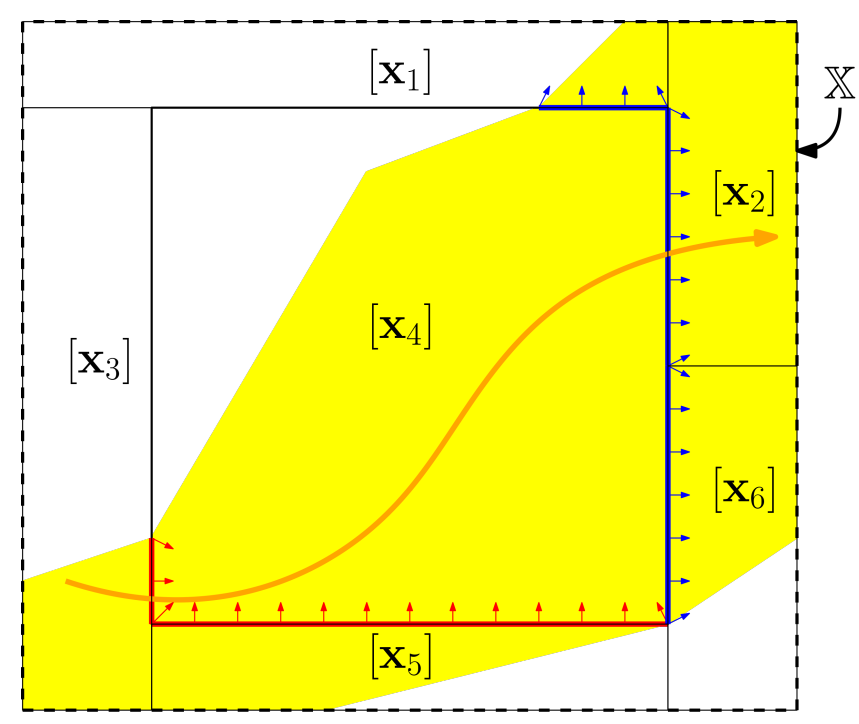

Figure 3: Illustration of a part of a maze where a possible trajectory is shown in orange. The $\left\{\left[\mathbf{x}_{i}\right]\right\}_{i \in\{1, \ldots, 6\}}$ form a paving of $\mathbb{X}$.

- A flow contractor that contracts the polygons and doors consistently with f,

- A continuity contractor that contracts the polygons and doors of a box $\mathbf{p}$ according to its neighbors.

Figure 3 illustrates the principle of the continuity and the flow contractors. The blue output door of [a] is contracted according to the red input door of [b] using the continuity contractors. The polygons associated to [a] and [b] are then contracted using the flow contractor. This last contraction could have then contract the doors of each boxes but this is not the case in the example of Figure 3.

Algorithm. To combine the outer and the inner approach we will use two mazes, one for the inner and one for the outer approximation. The inner approximation is very similar to the outer method as it works on a complementary approach as described in [17. We propose the following algorithm to bracket the largest invariant set:

1. Initialize the two mazes, one for the inner and one for the outer approximation, with the initial condition which means that the doors and polygons which correspond to the initial condition are set consistently with it;

2. Apply the contractors on each maze until a fix point is reached for the inner and the outer maze; 

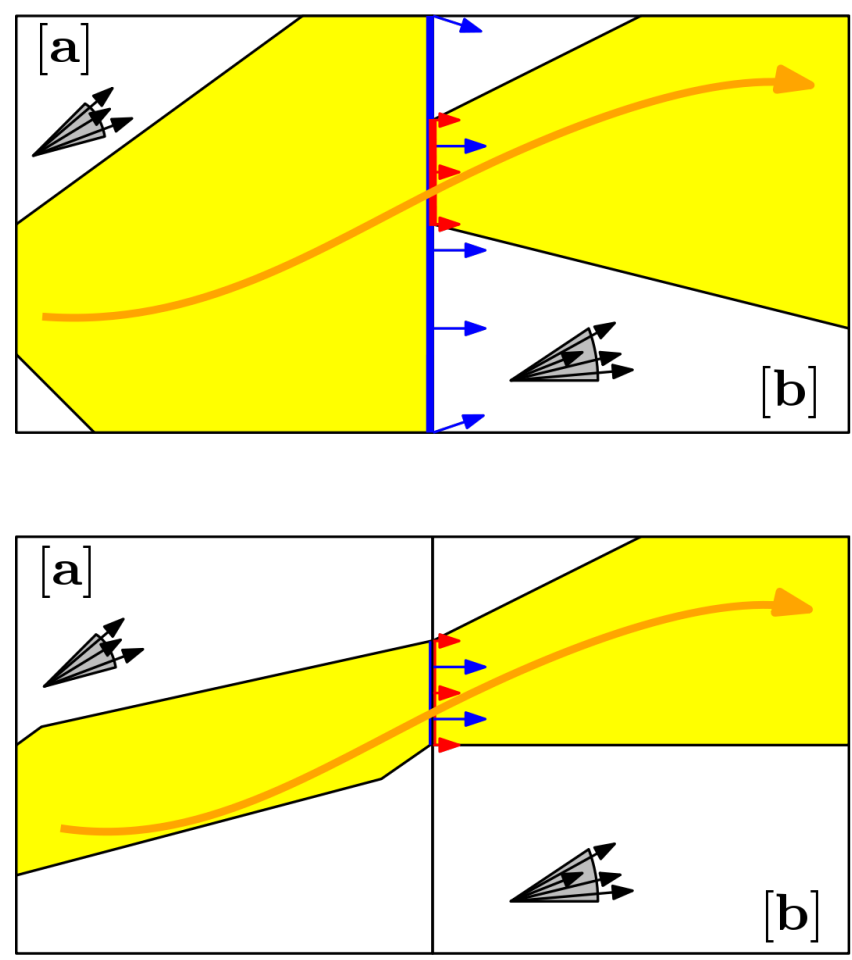

Figure 4: Illustration of the flow and the continuity contractors for a maze. The maze at the top is before the contraction and the bottom maze is after applying the contractors. The gray cones represent the union of all possible trajectories consistent with $\mathbf{f}$ in the box. This cone can be computed using interval computation. The orange curve is a possible path enclosed by the maze. 
3. Show the result OR bisect all boxes where the inner and the outer polygons are not empty, and go to Step 1.

Test-cases

We will now test our algorithm on two test-cases. All computations were conducted on a single processor i5-3230M@2.60GHz for all test-cases.

Test-case 1. Van der Pol system. Consider the system described by the state equation:

$$
\left\{\begin{array}{l}
\dot{x}_{1}=x_{2} \\
\dot{x}_{2}=\left(1-x_{1}^{2}\right) \cdot x_{2}-x_{1}
\end{array}\right.
$$

The initial vector satisfies $\mathbf{x}_{0} \in\left[\mathbf{x}_{0}\right]=[-4,-3] \times[3,4]$. Our algorithm is able to characterize the inner and outer approximation of the forward reachable set $\mathbb{F}_{\left[\mathbf{x}_{0}\right]}^{+}$as shown in Figure 3 The algorithm was stopped after 17 steps which takes $8 \mathrm{~s}$. The search set, which also corresponds to the frame box of the figure, corresponds to $\mathbb{X}=[-6,6] \times[-6,6]$.

We have tested this example on the solver CAPD 23] which is the state of the art of interval integration. It works with a guaranteed Lagrangian approach. We start the simulation at $t=0$, the solver rapidly diverges and stops computing at $t=0.57$ with the answer $\mathbf{x} \in[-722.795,716.825] \times[-3.24089 \mathrm{e}+8,3.24089 \mathrm{e}+8]$. This is due to the fact that the initial box is large.

Test-case 2. Car on the hill system [12]. Consider the system described by the state equation:

$$
\left\{\begin{array}{l}
\dot{x}_{1}=x_{2} \\
\dot{x}_{2}=-9.81 \sin \left(\frac{\frac{1.1}{1.2} \sin \left(x_{1}\right)-1.2 \sin \left(1.1 x_{1}\right)}{2}\right)-0.7 x_{2}+2.0 .
\end{array}\right.
$$

and the initial box $\left[\mathbf{x}_{0}\right]=[-1,1] \times[-1,1]$ and a search set $\mathbb{X}=[-2,13] \times[-6,6]$. As in [18] we are able to characterize the outer approximation of the set. The main improvement is to be able to characterize an inside and outside approximation in the same time: the need to bisect boxes is then reduced. Indeed, the bisection effort of the algorithm is now focused on the boundary between the inner and the outer approximation whereas on large outer approximation zones that were bisected in previous works [18. The result is shown on Figure 3 . The algorithm was stopped after 18 steps which takes $30 \mathrm{~s}$.

One of the drawback of our algorithm is that when trajectories leave the search setX $\mathbb{X}$, we are not able to conclude if that they will not come back later. This is the case on Figure 3 where trajectories can leave the search set $\mathbb{X}$ on its right top part.

The next section shows how this problem can be addressed, at least for some specific cases, by considering unbounded search boxes. 


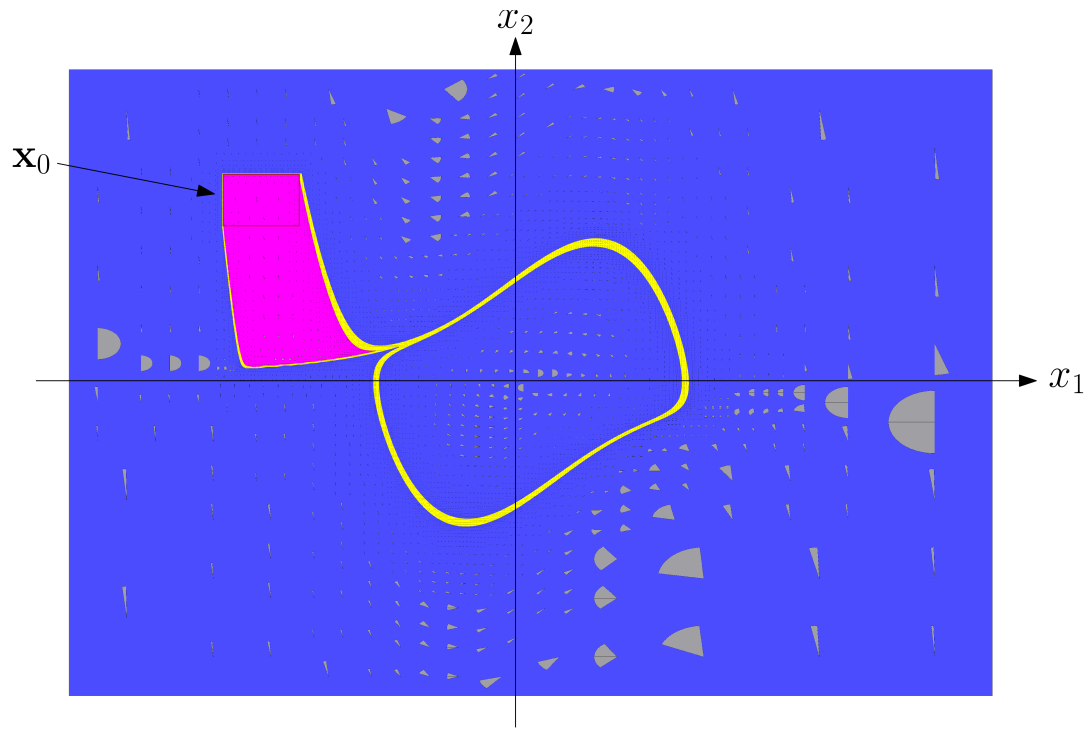

Figure 5: Bracketing $\mathbb{F}_{\left[\mathbf{x}_{0}\right]}^{+}$for the Van der Pol system. The magenta set corresponds to the inner approximation of $\mathbb{F}_{\left[\mathbf{x}_{0}\right]}^{+}$and the union of the yellow and magenta set to the outer approximation of $\mathbb{F}_{\left[\mathbf{x}_{0}\right]}^{+}$. The initial box $\left[\mathbf{x}_{0}\right]$ is painted in red and the blue area corresponds to the set where there is no solution. Gray cones correspond to the union of all directions of the vector field in each boxes. 


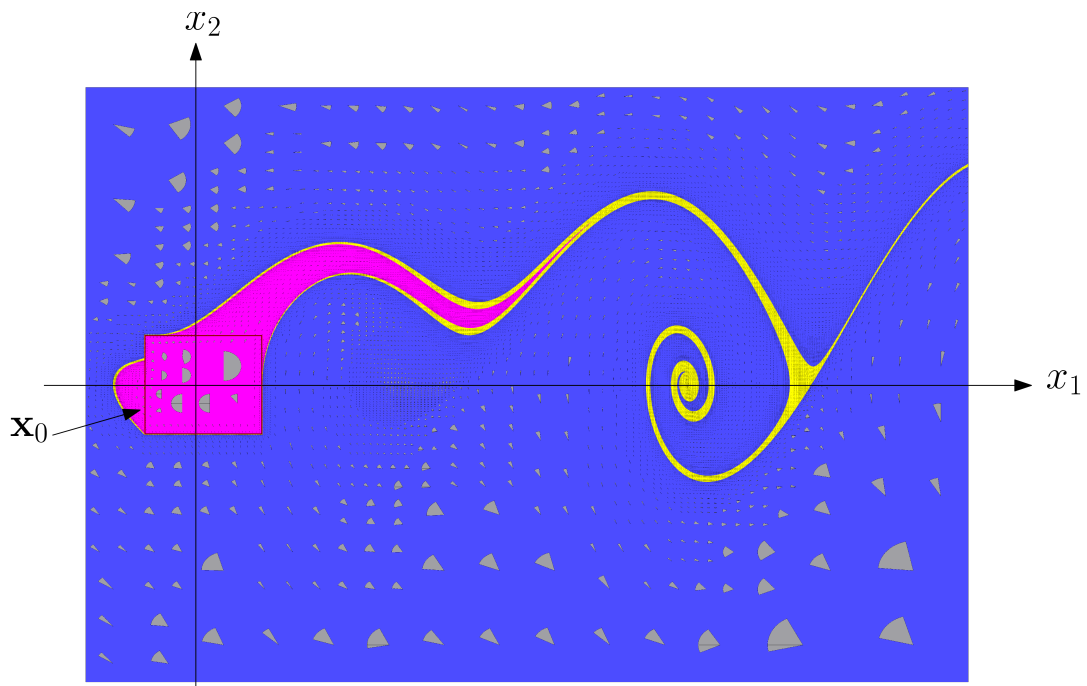

Figure 6: Bracketing $\mathbb{F}_{\left[\mathbf{x}_{0}\right]}^{+}$for the Car on the hill system. The color code is the same as Figure 3 Now, since the boundary of the approximations intersets the boundary of the initial box $\left[\mathbf{x}_{0}\right]$, we cannot guarantee anymore that the blue area is outside $\mathbb{F}_{\left[\mathbf{x}_{0}\right]}^{+}$.

\section{Dealing with infinity}

The previous section explained how to compute inner and outer approximations of $\operatorname{Inv}^{+}(\mathbf{f}, \mathbb{X})$ where $\mathbb{X}$ is a bounded box. Now, to characterize a positive graph, the set $\mathbb{X}$ is not bounded anymore and the abstract interpretation approach will need to handle unbounded intervals. Solving equations on an infinite intervals can still be performed combining interval constraint propagation with the generalized interval arithmetic [8]. In this arithmetic an interval is a connected subset of the extended real numbers, the set of all real numbers augmented with $-\infty$ and $+\infty$. For instance, with this arithmetic,

$$
\begin{aligned}
& & {[-\infty, \infty]^{2} \cap\left([-\infty, 2]^{3}-[-\infty, \infty]^{2}\right) } \\
= & & {[0, \infty] \cap([-\infty, 8]-[0, \infty]) } \\
= & & {[0, \infty] \cap[-\infty, 8]=[0,8] . }
\end{aligned}
$$

Similar results could have been obtained and probably made more accurate, using the arithmetic of infinity [22].

Example. To illustrate the propagation process, consider the three following equations

$$
\left\{\begin{array}{cc}
\left(C_{1}\right): & y=x^{2} \\
\left(C_{2}\right): & x y=1 \\
\left(C_{3}\right): & y=-2 x+1 .
\end{array}\right.
$$

Using interval propagation, we want to prove that this system has no solution. To each of the variables, we assign the domain $[-\infty, \infty]$. Then, we contract the 


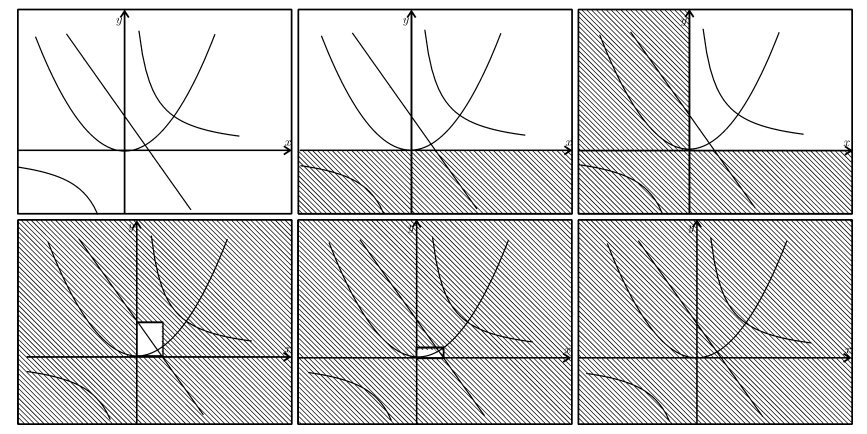

Figure 7: Illustration of the propagation procedure

domains with respect to the constraints in the following order: $C_{1}, C_{2}, C_{3}, C_{1}, C_{2}$ and we get empty intervals for $x$ and $y$. A geometric interpretation of the propagation is given on Fig. 7.

The resulting interval computation is as follows:

$$
\begin{aligned}
\left(C_{1}\right) \Rightarrow y \in & {[-\infty, \infty]^{2}=[0, \infty] } \\
\left(C_{2}\right) \Rightarrow & x \in 1 /[0, \infty]=[0, \infty] \\
\left(C_{3}\right) \Rightarrow y \in & {[0, \infty] \cap((-2) \cdot[0, \infty]+1) } \\
& =[0, \infty] \cap([-\infty, 1])=[0,1] \\
& x \in[0, \infty] \cap(-[0,1] / 2+1 / 2)=\left[0, \frac{1}{2}\right] \\
\left(C_{1}\right) \Rightarrow y \in & {[0,1] \cap[0,1 / 2]^{2}=[0,1 / 4] } \\
\left(C_{2}\right) \Rightarrow \quad x \in & {[0,1 / 2] \cap 1 /[0,1 / 4]=\emptyset } \\
y \in & {[0,1 / 4] \cap 1 / \emptyset=\emptyset . }
\end{aligned}
$$

until a steady box (also called the fixed point) is reached.

Bisection. To apply the maze method, we need to bisect intervals that can be unbounded. Now, in the interval literature, only bounded intervals of $\mathbb{R}$ are bisected and the cut point is the center. For instance, the interval $[-1,3]$ is bisected into $[-1,1]$ and $[1,3]$. Here, to bisect unbounded intervals we propose to fix a support interval $[a, b]$ and to never allow any bisections outside this support interval. For instance the interval $[-\infty, \infty]$ is bisected into $[-\infty, a]$ and $[a, \infty]$; the interval $[a, \infty]$ is bisected into $[a, b]$ and $[b, \infty]$. But the intervals $[-\infty, a]$ and $[b, \infty]$ are never bisected. They are considered as 'too small'.

\section{Bracketing the largest invariant set with unbounded search set}

Method. We are now able to build mazes with infinite boxes. We will use $2 \cdot n$ extra unbounded boxes around a search set $\mathbb{Y}$ to pave $\mathbb{R}^{n}$. Figure 5 shows an example of an unbounded set $\mathbb{X}$ composed of the bounded search set $\mathbb{Y}$ and extra unbounded boxes $\left\{\left[\mathbf{b}_{1}\right], \ldots,\left[\mathbf{b}_{k}\right]\right\}$ such as $\mathbb{X} \in\left\{\mathbb{Y} \cup\left[\mathbf{b}_{1}\right] \cup \ldots \cup\left[\mathbf{b}_{k}\right]\right\}$. We 


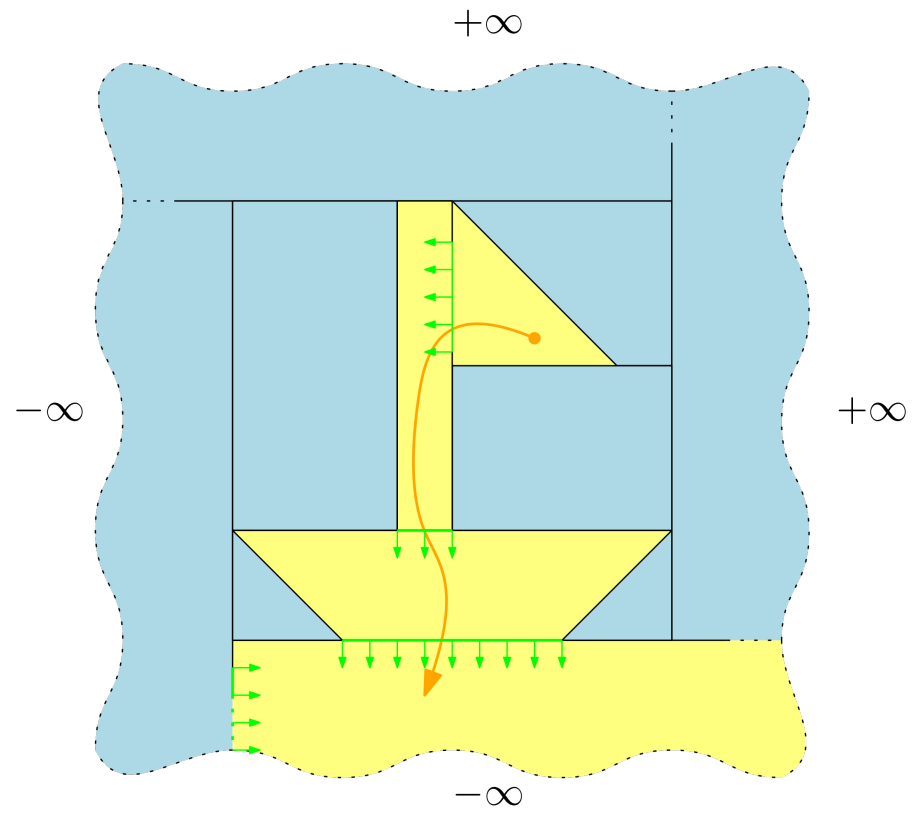

Figure 8: Illustration of the maze with unbounded boxes. The orange path corresponds to a trajectory that reaches the bottom infinity box but stays inside it according to the green doors.

can use the same algorithm from section 3 as we are able to apply contractors on unbounded domains. Indeed, we are able to compute the cone of all direction vectors inside unbounded boxes to apply the flow contractor and able to apply the continuity contractor.

In this part, we will illustrate the method of infinite extension to compute $\mathbb{F}_{\left[\mathbf{x}_{0}\right]}^{+}$or $\mathbb{G}_{\left[t_{0}\right],\left[\mathbf{x}_{0}\right]}^{+}$when the search box $\mathbb{X}$ is unbounded.

Test-case 3. Sinusoidal system. Consider the system described by the state equation:

$$
\dot{x}=-\sin x+e
$$

with the initial condition $t_{0} \in[0.3,0.5]$ and $x_{0} \in[-0.5,0.5]$ and where $e \in[-0.1,0.1]$. We choose $\mathbb{X}=[0,5] \times[-2,2]$. As illustrated by Figure 5 trajectories leaves the search set on the right part of the figure only and that they cannot come back on the left part of the figure. This result is consistent with the state equation where time always increases and forces trajectories to not come back on the right of the figure. If we want to study more globally this system, we now know that it is only necessary to extend the search set on the 


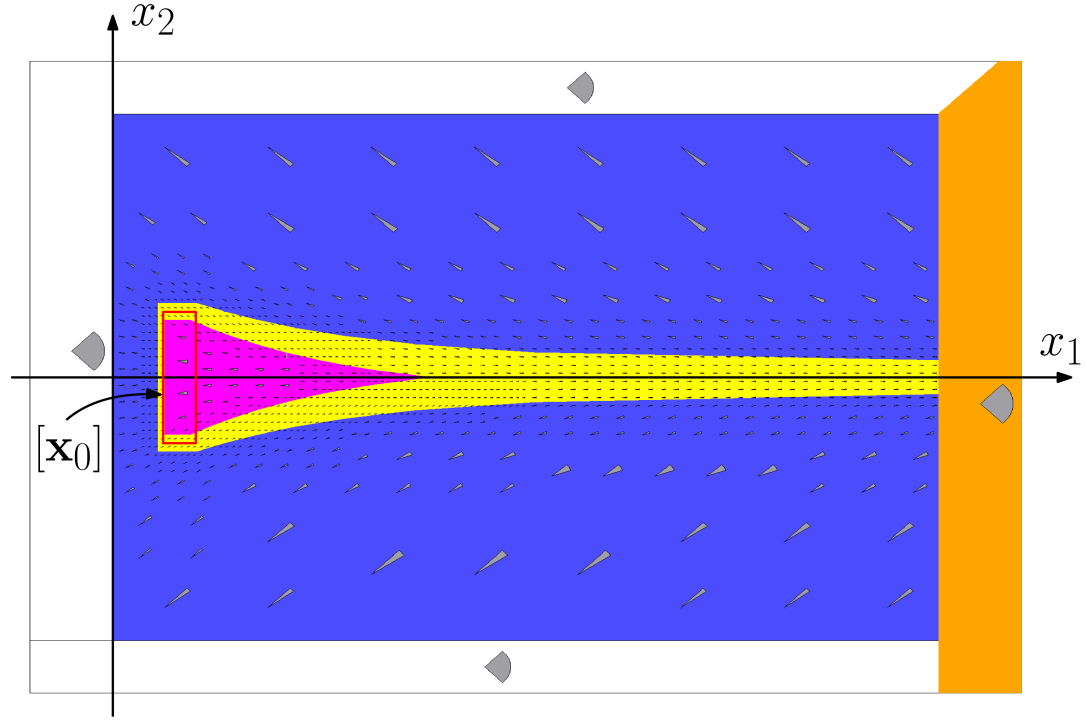

Figure 9: Bracketing the positive graph of the sinusoidal system with an unbounded search set. The color code is the same as Figure 3 The surrounding boxes of the blue frame are the infinite boxes where their polygon is colored in orange.

right part of the figure. The algorithm was stopped after 10 steps which takes $0.7 \mathrm{~s}$.

Test-case 4. Car on the hill system. We now take back test-case 2 with the example of the Car on the hill system where the bounded search set was $\mathbb{X}=[-2,13] \times[-6,6]$. Figure 5 shows the result of the algorithm with infinite boxes. Unlike the result of test-case 2, it was not possible here to contract the outer approximation (no blue zones). In fact, we have seen that trajectories were able to leave the bounded search set on the right part of Figure 3 in section 3. According to the cones of all possible trajectories of the unbounded boxes, theses trajectories can re-enter inside the search-set (see the right cone). This is why we cannot have a better result of the outer approximation. A larger zone should be studied: an extend of the search space on the right part of the figure is required to improve the result. The algorithm was stopped after 18 steps which takes $190 \mathrm{~s}$.

Test-case 4-bis. Figure 5 shows the result of our algorithm on the Car on the hill system with a larger bounded search set $\mathbb{X} \in[-2,17] \times[-6,6]$. We can see that no trajectories leave the bounded search set: this is why there is an empty polygon in all of the infinite boxes and why we were able to contract the outer approximation. All the trajectories stay inside $\mathbb{X}$. The algorithm was 


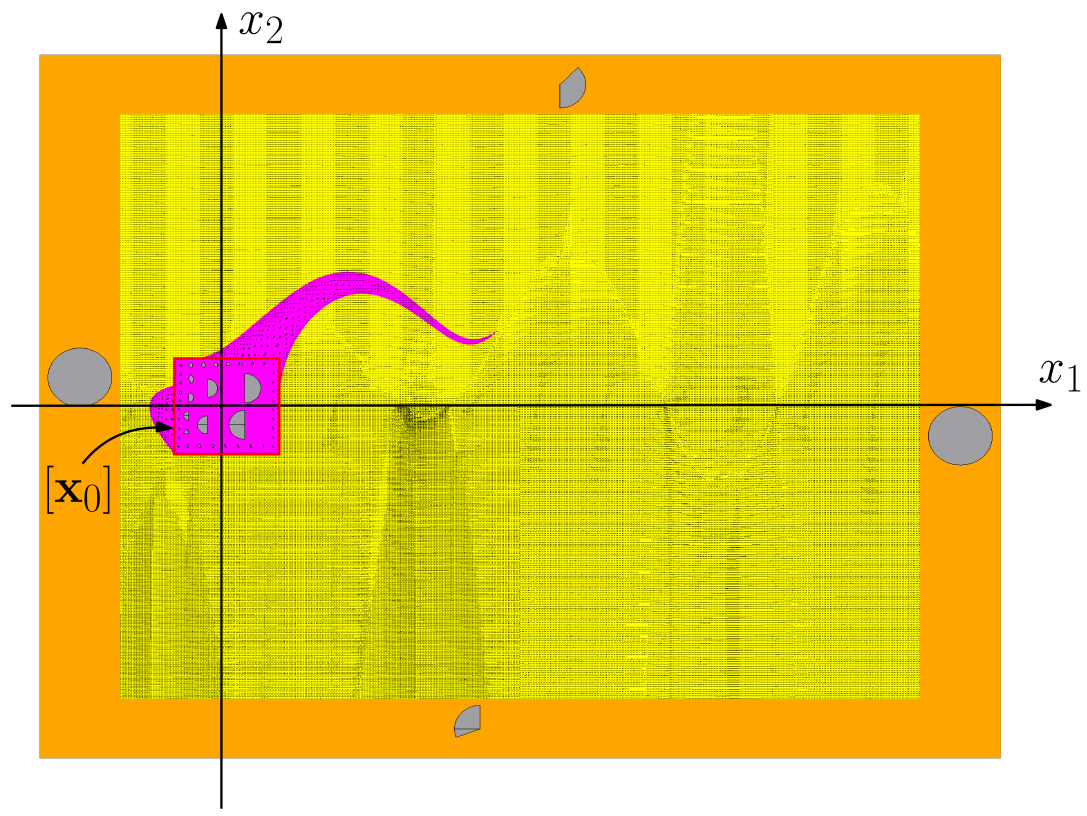

Figure 10: Bracketing the forward reachable set of the Car on the hill system with an initial condition and an infinite search space. Infinite boxes are orange colored and gray circles mean that all directions might be possible inside the box. 


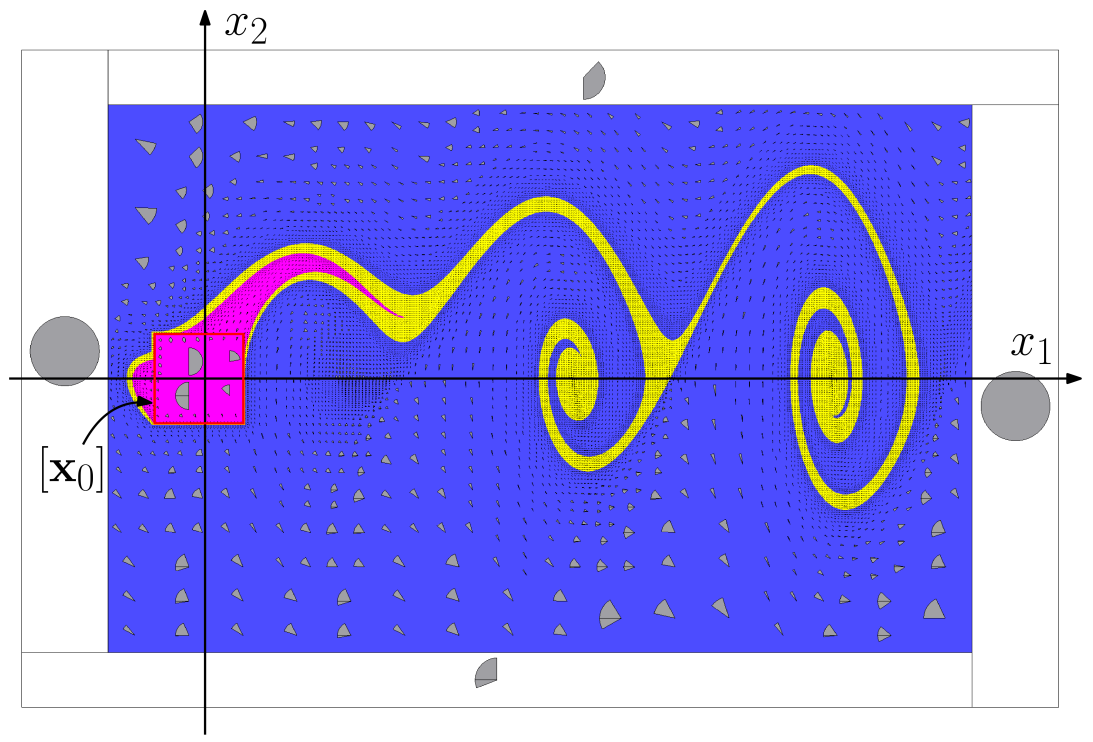

Figure 11: Bracketing the forward reachable set of the Car on the hill system with an initial condition and an infinite search space. The search space was set larger than Figure 5

stopped after 16 steps which takes $14 \mathrm{~s}$.

\section{Conclusion}

In this paper, we have proposed a new approach to bracket the solutions of an ordinary differential equation with uncertain initial conditions. We have shown that this problem can be seen as an enclosure of the positive invariant set of the system. By using mazes to work with trajectories and by using tools from constraint programming and abstract interpretation theory, we have been able to propose an algorithm to compute some inner and the outer approximations of the solution of our problem. Finally we have extended the use of mazes to unbounded set which increases the number of problems that our method can deal with. The method was also tested on several test-cases.

Our method needs to bisect the state space, contrary to most interval integration methods making our approach Eulerian. On the one hand, this implies an exponential increases of the computational complexity with respect to the dimension of the state space. This makes our method impracticable with high dimensional systems.

In a near future, we plan to extend our approach to dealing with hybrid systems [11] when jumps could occur with respect to some guard conditions. 


\section{Acknowledgment}

This work has been supported by the French Government Defense procurement and technology agency (DGA).

[1] I. Araya, G. Trombettoni, and B. Neveu. A Contractor Based on Convex Interval Taylor. In Proc. of CPAIOR, pages 1-16, LNCS 7298, Springer, 2012 .

[2] E. Asarin, T. Dang, and A. Girard. Reachability analysis of nonlinear systems using conservative approximation. In Hybrid Systems: Computation and Control, pages 20-35. Springer Berlin Heidelberg, 2003.

[3] Franco Blanchini and Stefano Miani. Set-Theoretic Methods in Control. Springer Science \& Business Media, October 2007.

[4] G. Chabert and L. Jaulin. Contractor Programming. Artificial Intelligence, 173:1079-1100, 2009.

[5] P. Collins and A. Goldsztejn. The Reach-and-Evolve Algorithm for Reachability Analysis of Nonlinear Dynamical Systems. Electronic Notes in Theoretical Computer Science, (223):87-102, 2008.

[6] J Alexandre dit Sandretto and Alexandre Chapoutot. Validated explicit and implicit runge-kutta methods. Reliable Computing, 22:79, 2016.

[7] E. Goubault, O. Mullier, S. Putot, and M. Kieffer. Inner approximated reachability analysis. In Proceedings of the 17th international conference on Hybrid systems: computation and control, HSCC '14, pages 163-172, Berlin, Germany, 2014.

[8] E. R. Hansen. A generalized interval arithmetic. In K. Nickel, editor, Interval Mathematics 1975, pages 7-18. Springer-Verlag, 1975.

[9] M. Hladik. Enclosures for the solution set of parametric interval linear systems. Int. J. Appl. Math. Comput. Sci., 22(3):561-574, 2012.

[10] Shahab Kaynama, John Maidens, Meeko Oishi, Ian M. Mitchell, and Guy A. Dumont. Computing the Viability Kernel Using Maximal Reachable Sets. In Proceedings of the 15th ACM International Conference on Hybrid Systems: Computation and Control, HSCC '12, pages 55-64, New York, NY, USA, 2012. ACM.

[11] Michal Konečnỳ, Walid Taha, Ferenc A Bartha, Jan Duracz, Adam Duracz, and Aaron D Ames. Enclosing the behavior of a hybrid automaton up to and beyond a zeno point. Nonlinear Analysis: Hybrid Systems, 20:1-20, 2016. 
[12] M. Lhommeau, L. Jaulin, and L. Hardouin. Capture basin approximation using interval analysis. International Journal of Adaptive Control and Signal Processing, 25(3):264-272, March 2011.

[13] Mehdi Lhommeau, Luc Jaulin, and Laurent Hardouin. Inner and outer approximation of capture basin using interval analysis. In ICINCO-SPSMC, pages $5-9,2007$.

[14] A. K. Mackworth. Consistency in networks of relations. Artificial Intelligence, 8(1):99-118, February 1977.

[15] John N. Maidens, Shahab Kaynama, Ian M. Mitchell, Meeko M. K. Oishi, and Guy A. Dumont. Lagrangian methods for approximating the viability kernel in high-dimensional systems. Automatica, 49(7):2017-2029, July 2013.

[16] S. Le Menec. Linear Differential Game with Two Pursuers and One Evader. In M. Breton and K. Szajowski, editors, Advances in Dynamic Games, volume 11, pages 209-226, 2011.

[17] T. Le Mézo, L. Jaulin, and B. Zerr. Inner approximation of a capture basin of a dynamical system. In Abstracts of the 9th Summer Workshop on Interval Methods SWIM'2016. Lyon, France, June 19-22, 2016.

[18] T. Le Mézo, L. Jaulin, and B. Zerr. An interval approach to solve an initial value problem. AIP Conference Proceedings, 1776(1), 2016.

[19] T. Le Mézo, L. Jaulin, and B. Zerr. An interval approach to compute invariant sets. IEEE Transactions on Automatic Control, PP(99):1-1, 2018.

[20] Nedialko S Nedialkov, Kenneth R Jackson, and George F Corliss. Validated solutions of initial value problems for ordinary differential equations. Applied Mathematics and Computation, 105(1):21-68, 1999.

[21] N. Ramdani and N. Nedialkov. Computing Reachable Sets for Uncertain Nonlinear Hybrid Systems using Interval Constraint Propagation Techniques. Nonlinear Analysis: Hybrid Systems, 5(2):149-162, 2011.

[22] Yaroslav D. Sergeyev. Arithmetic of Infinity. Kindle Edition, 2003.

[23] Irmina Walawska and Daniel Wilczak. An implicit algorithm for validated enclosures of the solutions to variational equations for $\{\mathrm{ODEs}\}$. Applied Mathematics and Computation, 291:303 - 322, 2016. 